Lepr Rev (1989) 60, 257-262

\title{
Editorial
}

\section{THE USE OF HISTOPATHOLOGY IN LEPROSY DIAGNOSIS AND RESEARCH}

\section{Introduction}

The way we think and talk about leprosy, using the terms 'tuberculoid' and 'lepromatous', is histopathologically orientated. In the most widely used system of leprosy classification - the Ridley-Jopling classification-histology plays the major role. ${ }^{1}$ In clinical practice, there is no doubt that the histological assessment by an experienced pathologist of a representative skin (and/or nerve) biopsy and enumeration of bacilli provides information as significant as that derived from the clinical examination of a patient.

Only a small proportion of the estimated $10+$ million patients with leprosy have been biopsied. The current uses of tissue biopsy fall into two broad categories: diagnosis of leprosy and leprosy reactions; and research into inflammatory processes. Some aspects, particularly those with practical applications, are discussed here.

\section{Diagnosis of leprosy-early disease}

Early diagnosis of leprosy is a prerequisite for control as well as for effective therapy. Epidemiologically, the total number of cases of leprosy needs to be established for a region, and much underestimation may ensue from the poor detection of early cases. There is no independent 'gold standard' for the diagnosis of leprosy. Neither serological nor skin tests have sensitivity and specificity high enough to be useful in confirming or eliminating all suspect cases of leprosy. How usef ul is histopathology? The two cardinal histological features of leprosy are appropriate patterns of inflammation involving certain sites in skin (e.g. nerves), and the demonstration of acid-fast bacilli (AFB) in appropriate sites. The degree of confidence that individual pathologists place in a diagnosis of early leprosy is variable.

The importance of accurate histological assessment is emphasized by the widespread poor performance of slit-skin smear bacteriology: low densities of AFB in skin are underestimated or missed entirely, so that cases are missed as well as being misclassified. ${ }^{2}$

Only one systematic study has been published on the performance of pathologists on leprosy. Three experienced histopathologists examined the same 143 skin biopsies from leprosy suspects in Malawi, where $95 \%$ of leprosy is paucibacillary. ${ }^{3}$ The results appear disturbing. The proportions of biopsies classed as showing strong or definite evidence of leprosy ranged from $39 \%$ to $58 \%$. The proportions of biopsies thought to be 'possibly leprosy' ranged from $11 \cdot 5 \%$ to $38 \cdot 5 \%$, reflecting an unexpectedly large variation in degree of uncertainty. Nonetheless, considering only the 82 cases that were clinically thought certain to be leprosy, the histopathologists did agree in $63 \%$ to $83 \%$ of cases. ${ }^{4}$ Much of the discrepancy in diagnostic certainty derived from differences in the interpretation of nerve involvement by granulomatous or nongranulomatous inflammation. AFB were uncommonly detected in the biopsies from this population. 
The category 'indeterminate' was used by one pathologist in $1.5 \%$ of cases, yet in $21.5 \%$ of cases by another. Evidently there is much dissent on what is meant by 'indeterminate'. ${ }^{1}$ It has to be said that the material used in this study, being derived from intensive population surveys for leprosy, is much 'earlier' than is generally seen in biopsies from patients who self-present; the great majority of these biopsies had granuloma fractions of $<10 \%$ (see below).

If pathologists cannot consistently diagnose early, indeterminate leprosy, are there alternative methodologies? Specifically, since the histological evaluation of inflammatory patterns cannot be automated, could tissues be better analysed for the presence of Mycobacterium leprae and thus support a diagnosis of leprosy?

Immunocytochemistry using polyclonal rabbit anti-BCG antibodies is certainly effective in demonstrating $M$. leprae antigen in multibacillary tissues, even when the bacilli are reduced to debris or are invisible on acid-fast stains. ${ }^{5}$ However, convincing demonstration of antigen where AFB density is effectively zero has not been shown. Monoclonal antibodies against phenolic glycolipid-1 (PGL-1) and various defined antigens of $M$. leprae have been used. With fluorescent labelling, anti PGL-1 antibodies detected positive staining in 7/19 cases of indeterminate leprosy, only one of which was positive by acid-fast staining. ${ }^{6}$ Immunoperoxidase staining with a panel of monoclonals showed occasional deposits of the $36 \mathrm{kd}$ antigen in macrophages of tuberculoid leprosy skin biopsies where AFB were not ordinarily visible. ${ }^{7}$ Immunogold staining in tissues processed for electron microscopy demonstrates PGL-1 well in bacillary capsular material, but it has not been tried on paucibacillary cases. ${ }^{8}$

Whilst techniques of in situ DNA hybridization have great sensitivity in detecting the DNA of viruses in tissues, it has not been applied to the detection of mycobacterial DNA. Theoretically, it is unlikely to be useful since it can only label and bring out the quantity of bacillary DNA that is already present.

However, the recent technique of polymerase chain reaction (PCR) amplifies DNA nucleotide sequences in tissues to bring out minute quantities: the results are not read on tissue sections but as bands on Southern blot gels. The sensitivity that can be achieved is impressive. With a suspension of $M$. leprae used as the substrate, the detection limit appears to be 1-10 bacilli. Using homogenates of armadillo livers as substrate, $10^{7}$ bacilli per gram were detectable. ${ }^{9}$ How the technique of PCR will work using sections from fixed human tissues is under investigation.

Of these alternative techniques for detecting small numbers of AFB in tissues, the immunocytochemical methods are, in our opinion, difficult to interpret and are not reliable in early diagnosis; often there is much background staining. The PCR may be a breakthrough in sensitivity (it must be remembered that acid-fast stains can theoretically detect positive bacilli at a concentration of about 500 per cc of tissue), but it is technically demanding, and like the other techniques, will be difficult to perform adequately in those developing countries where leprosy is endemic. Finally, histologically, the presence of an acid-fast bacillus in sections is convincing when it is located in an appropriate site and when the associated inflammation, however slight, is consistent with our experience of leprosy. It remains to be seen whether the apparent identification of $M$. leprae DNA in tissue by PCR is perse convincing evidence of leprosy in a suspect case, or whether effort might not be more profitably put into the examination of more sections stained by classical techniques.

\section{Diagnosis of leprosy—more advanced disease}

Histologically, multibacillary leprosy presents little problem once it has been considered. Foamy macrophages containing bacillary debris may be considered xanthomas, but immunocytochemistry and Grocott methods will demonstrate the mycobacterial nature of the inflammation. ${ }^{5}$

However, granulomatous dermatitis without apparent AFB has a large differential diagnosis: sarcoidosis, granuloma annulare, granuloma multiforme, syphilis, leishmaniasis and other mycobacterioses are the main problems. The new techniques of bringing out AFB or their DNA are 
discussed above. The detection of nerve involvement is important, and the staining combination of periodic acid-ethanol gelatine and methenamine silver can show both bacterial cell walls and myelin in the same section, given that AFB are present. ${ }^{10}$ Apart from these, the only new technique is the immunocytochemical demonstration of dermal nerves using antibodies to S100 protein. This can show single Schwann cells and may confirm endoneurial nerve involvement or destruction by granulomas, so confirming leprosy. ${ }^{11}$ Like most aspects of leprosy histology, this has not undergone a formal trial of sensitivity and applicability. Whilst the nonhistological methods of diagnosis of lesions confusable with leprosy constantly improve, it seems unlikely that systematic alternatives to good histological sections and experience of leprosy pathology will develop in the forseeable future.

In the comparability study quoted earlier, ${ }^{4}$ there was reasonably good concordance between the pathologists on classification of determined leprosy lesions (only early leprosy was problematic). Further studies of pathologists' performance are now continuing, with intraobserver as well as interobserver variation being investigated. The degree to which different types of leprosy prevail in different parts of the world may have, uncomfortably, much to do with the variation between observers as well as that between the patients.

When patients present themselves, the problem of histopathological diagnosis of leprosy is considerably less. Personal observations (SBL) of biopsies from 508 near-consecutive patients suspected of having leprosy seen in the Marie Adelaide Leprosy Centre, Karachi, indicate that $89 \%$ were readily diagnosable as leprosy. Of those, $2 \cdot 2 \%$ were indeterminate (nongranulomatous) with positive $\mathrm{AFB}$, and the rest were determined paucibacillary or multibacillary leprosy. Only $6 \%$ were possibly but not certainly indeterminate leprosy (i.e. had no AFB in sections), and 5\% were diagnosed as disease other than leprosy. In summary, the histopathological difficulties in establishing leprosy increase markedly when active surveying for early lesions is undertaken. ${ }^{3}$

\section{Neuritis}

It has become evident that the bacillary indices in skin and peripheral nerve biopsies taken from patients at the same time are discrepant in many cases (possibly $50 \%$ ). ${ }^{12-14}$ The nerves may contain a bacterial density up to one thousand $(\log 3)$ times that of skin. At least two implications follow. The classification of a patient as paucibacillary or multibacillary is according to skin bacterial index; but it would appear that if nerves are assessed routinely by biopsy, more patients would be classed as multibacillary. The regimes of multidrug therapy (MDT) may need to be considered accordingly. Secondly, the higher neural bacillation supports the impression that many relapses of leprosy commence in nerves. ${ }^{12}$ Long-term post-MDT studies with particular attention to nerve pathology are needed.

Immunocytochemical staining for neuropeptides in skin biopsies has demonstrated the fine terminal nerves that permeate the upper dermis and epidermis. In leprosy there is progressive reduction in their number through indeterminate, lepromatous and tuberculoid lesions, indicating that in even very early lesions, dermal nerves are being damaged. ${ }^{15}$ Future studies with these markers may provide evidence that a common location of AFB in early lesions, the apparently acellular subepidermal zone, may actually represent location within fine unmyelinated nerves. The patterns of regrowth of these dermal nerves after chemotherapy is of great interest.

\section{Cellular characterization of leprosy lesions}

Immunocytochemical staining techniques for inflammatory cell phenotypes are now a routine research procedure in leprosy. The earlier established patterns of T-cell subsets are confirmed, namely: $\mathrm{T}$-helper $(\mathrm{CD} 4+)$ : suppressor/cytotoxic $(\mathrm{CD} 8+)$ ratios are greater in tuberculoid lesions than in lepromatous; CD4 + lymphocytes are distributed throughout tuberculoid granulomas; 
CD8 + lymphocytes are restricted to the outer mantle of tuberculoid granulomas but are randomly distributed through lepromatous lesions. ${ }^{16,17}$ More subtle (but still controversial) differential patterns of helper/inducer cells, suppressor/inducer cells and cytotoxic versus suppressor CD8 + cells are also described. ${ }^{16}$ Such T-cell subset patterns in leprosy have stimulated similar examination of tuberculosis, ${ }^{18}$ leishmaniasis ${ }^{19}$ and sarcoidosis ${ }^{18}$ lesions: the differences that emerge in cell reactions between leprosy and these other granulomatous diseases will provide information on their pathogenetic mechanisms.

Immunocytochemical studies in leprosy are helping to unravel the nature of the immune defect in lepromatous leprosy, as well as the causes of leprosy reactions. The local production of inflammatory mediators such as the monokine interleukin-1 (IL-1), and the lymphokines interleukin-2 (IL-2) and interferon-gamma (IFN- $\gamma$ ) can be estimated. The lack of these two lymphokines in lepromatous lesions ${ }^{17,20}$ may underlie the inability of macrophages to kill and clear $M$. leprae bacilli (IFN- $\gamma$ is an activator of macrophages).

In situ hybridization probes for mediators such as IFN $-\gamma$ mRNA is providing deeper insights into the pathogenesis of leprosy reactions, by allowing the enumeration of IFN- $\gamma$-producing cells. For example, whilst in both reversal reactions and erythema nodosum leprosum (ENL) reactions the number of CD4 + T-cells in lesions increases, increased IFN- $\gamma$ production occurs in the former but not the latter reaction: ${ }^{17}$ hence the lack of effective clearance of bacteria and antigen in ENL. Administering intradermal IFN- $\gamma$ induces delayed hypersensitivity phenomena in lepromatous lesions so that the lesions locally upgrade. The local bacterial index is rapidly reduced by an average of $\log 1.21,22$ (Effective chemotherapy reduces bacterial load by only log 1 per annum.) Intradermal injection of IL-2 into lepromatous lesions has an even more powerf ul effect on bacillary load: the range of reduction is $5-1000$, with a mean of $100(\log 2) .{ }^{23}$ These in vivo experiments support the current views on the immune defects in lepromatous leprosy. Moreover, there are possible therapeutic implications if IL-2 injection, into the skin or parenterally, is safe. Recurrent ENL is associated with large, persistent deposits of $M$. leprae antigen. IL-2 therapy may be able to reduce this load systemically and so ameliorate the condition of these unfortunate patients.

In situ studies of further inflammatory mediators will follow, using both direct estimates of concentration by immunocytochemistry and estimates of potential production using probes for mRNA. Of great interest will be the analysis of tumour necrosis factor (TNF), a macrophage product whose release is stimulated by IFN- $\gamma$. It is already implicated in the necrosis of tuberculosis lesions as well as some of that disease's systemic effects. ${ }^{24}$ TNF may also be responsible for the necrosis seen in severe reversal reactions in leprosy.

\section{Global aspects of leprosy histopathology}

The provision of general histopathology services for many developing countries is poor or nonexistent and much biopsy material is sent to developed countries for assessment. ${ }^{25.26}$ For leprosy histopathology, even less reporting is done on site, with wholesale despatch of tissues from leprosy hospitals to a few centres in Europe and the USA. Lack of laboratory facilities and finance, the difficulty of maintaining technical standards, and lack of pathologists are the major reasons. Inevitably this means that local pathologists are less familiar with the nuances of leprosy than they could and should be. If histopathology is important in overall leprosy management, then this situation is to be deprecated.

\section{Conclusion}

The difficulty and lack of agreement in diagnosing early (paucibacillary) leprosy by histopathology is frustrating. There is no reason why the essentially subjective nature of histological observation 
and diagnosis should change in the future. Whether the newer techniques of detecting M. leprae DNA or antigens will facilitate early diagnosis is not yet known; if they can help in a research centre, will they be useful in more peripheral laboratories? The study of cell types and mediators operating in leprosy lesions tells us about pathogenetic immune mechanisms, and may have therapeutic spinoffs. The global provision of histopathology is woefully inadequate. For leprosy, its present vertical arrangement is unlikely to change in the near future, and many endemic countries will continue to be dependent on the pathological expertise of developed countries.

S B LuCAS AND D S Ridley

\author{
Department of Histopathology \\ University College and Middlesex School of Medicine \\ University Street \\ London WCIE 6JJ
}

\title{
References
}

1 Ridley DS. Pathogenesis of leprosy and related diseases. Wright, London, 1988.

2 Georgiev GD, McDougall AC. The bacteriological examination of slit-smears in leprosy control programmes using multiple drug therapy; a plea for radical changes in current operational methodology. Ind J Lep, 1988; 59: 373-85.

${ }^{3}$ Ponnighaus JM, Fine PEM. Leprosy in Malawi 1. Sensitivity and specificity of the diagnosis and the search for the risk factors in leprosy. Trans $R$ Soc Trop Med Hyg, 1988; 82: 803-9.

${ }^{4}$ Fine PEM, Job CK, McDougall AC, Meyers WM, Ponnighaus JM. Comparability among histopathologists in the diagnosis and classification of lesions suspected of leprosy in Malawi. Int J Lepr, 1986; 54: 614-25.

5 Ridley MJ, Ridley DS. The immunopathology of erythema nodosum leprosum: the role of immune complexes. Lepr Rev, 1983; 54: 95-107.

${ }^{6}$ Huerre M, Desforges S, Bobin P, Ravisse P. Demonstration of PGL-1 antigens in indeterminate leprosy patients: comparison with serological anti-PGL-1 levels. Acta Le pr, 1987; 7 (suppl 1): 125-7.

${ }^{7}$ Khanolkar S, MacKenzie C, Lucas S, Huusen A, Girdhar B, Katoch K, McAdam K. The identification of Mycobacterium leprae antigens in the tissues of leprosy patients using monoclonal antibodies. Int $J$ Lepr, 1989; 57: 652-8.

${ }^{8}$ Boddingius J, Dijkman HP. Immunogold labelling method for Mycobacterium leprae-specific phenolic glycolipid in glutaraldehyde-osmium-fixed and araldite-embedded leprosy lesions. J. Histochem Cytochem, 1989; 37: 455-62.

${ }^{9}$ Hartskeerl RA, de Wit MYL, Klatser PR. Polymerase chain reaction for the detection of Mycobacterium leprae. J Gen Microbiol, 1989; in press.

${ }^{10}$ Harada K, Suzuki K. Periodic acid-ethanol gelatine and methenamine silver for demonstrating $M$ ycobacterium leprae and myelin in peripheral nerve fibres of leprosy patients. Int J Lepr, 1986; 54: 84-7.

11 Fleury RN, Bacchi CE. S-100 protein and immunoperoxidase technique as an aid in the histopathologic diagnosis of leprosy. Int J Lepr, 1987; 55: 338-44.

12 Srinivasan H, Rao KS, Iyer CGS. Discrepancy in the histopathological features of leprosy lesions in the skin and peripheral nerve. Lepr Ind, 1982; 54: 275-82.

13 Nilsen R, Mshana RN, Negesse Y, Mengistu G, Kana B. Immunohistochemical studies of leprous neuritis. Lepr Rev, 1986; 57 (suppl 2): 177-87.

14 Ridley DS, Ridley MJ. Classification of nerves is modified by the delayed recognition of Mycobacterium leprae. Int J Lepr, 1986; 54: 596-606.

15 Karanth SS, Springall DR, Lucas S, Levy D, Ashby P, Levene MM, Polak JM. Changes in nerves and neuropeptides in skin from 100 leprosy patients investigated by immunocytochemistry. J Pathol, 1989; 157: $15-26$.

16 Modlin RL, Melancon-Kaplan J, Young SMM, Pirmez C, Kino H, Convit J, Rea TH, Bloom BR. Learning from lesions: patterns of tissue inflammation in leprosy. Proc Natl Acad Sci USA, 1988; 85: 1213-17.

17 Cooper CL, Mueller C, Sinchaisri T-A, Pirmez C, Chan J, Kaplan G, Young SMM, Weissman IL, Bloom BR, Rea TH, Modlin RL. Analysis of naturally occurring delayed-type hypersensitivity reactions in leprosy by in situ hybridisation. $J$ Exp Med, 1989; 169: 1565-81.

18 van Oord JJ, de Wolf-Peeters C, Fachetti F, Desmet VJ. Cellular composition of hypersensitivity-type granulomas: immunohistological analysis of tuberculous and sarcoidal lymphadenitis. Hum Pathol, 1984; 15: 559-65. 
19 Modlin RL, Tapia FJ, Bloom BR, Gallinoto ME, Castes M, Rondon AJ, Rea TH, Convit J. In situ characterisation of the cellular immune response in American cutaneous leishmaniasis. Clin Exp Immunol, 1985; 60: 241-8.

20 Volc-Platzer B, Stemberger H, Luger T, Radaskiewicz T, Wiedermann G. Def ective intralesional interf erongamma activity in patients with lepromatous leprosy. Clin Exp Immunol, 1988; 71: 235-40.

${ }^{21}$ Nathan CF, Kaplan G, Levis WR, Nusrat A, Witmer MD, Sherwin SA, Job CK, Horowitz CR, Steinman RM, Cohn ZA. Local and systemic eff ects of intradermal recombinant interferon-gamma in patients with lepromatous leprosy. $N$ Eng $J$ Med, 1986; 315: 6-15.

22 Samuel N, Grange JM, Samuel S, Lucas S, Owilli OM, Adalla S, Leigh IM, Navarette C. A study of the effects of intradermal administration of recombinant gamma-interferon in lepromatous leprosy patients. Lepr Rev, 1987; 58: 389-400.

${ }^{23}$ Kaplan G, Kiessling R, Teklemariam S, Hancock G, Sheftel G, Job CK, Converse P, Ottenhoff THM, BecxBleumink M, Dietz M, Cohn ZA. The reconstruction of cell-mediated immunity in the cutaneous lesions of lepromatous leprosy by recombinant interleukin-2. J Exp Med, 1989; 169: 893-907.

24 Rook GAW. Macrophage antimycobacterial mechanisms. Br Med Bull, 1988; 44: 611-23.

25 Dodds RE, Greene JF. Pathology in mission hospitals. Lab Med, 1988, 19: 177-81.

26 Hutt MSR, Spencer H. Histopathology services for developing countries, Br Med J, 1982; 285: 1327-9. 